\title{
The effects of extinction of food-reinforced responding on schedule-induced polydipsia*
}

\author{
ERNEST PONICKI† and TRAVIS THOMPSON \\ Psychiatry Research Unit, University of Minnesota, Minneapolis, Minn. 55455
}

The polydipsic behavior of three male albino rats was studied under conditions of extinction of a fixed-interval 128-sec food reinforcement inducing schedule (Ext 1). The inducing schedule was then reinstated and the chronic extinction procedure repeated (Ext 2). During Day 1 of Ext 1, water responding fell markedly and remained virtually unchanged during Day 2. On the third day, water responding declined to 1/3-1/9 of the Day 1 or Day 2 level. Ext 2 differed from Ext 1 in that fewer total water responses were emitted, responding fell sharply on Day 2, and a generally lower level of water responding occurred on Day 3. Spontaneous recovery of polydipsia was consistently observed.

Falk (1961) initially reported the polydipsic water intake by rats that were maintained on a variable-interval schedule of food-reinforced leverpressing. The generality of the phenomenon has been extended to a variety of response-dependent and response-independent schedules of food presentation: fixed interval (Falk, 1966a, b), fixed time (Segal et al, 1965), variable time (Hawkins, 1967 ), fixed ratio (Schaeffer \& Diehl, 1966), and second-order fixed interval (Rosenblith, 1970). There have been several studies concerning the extinction of the polydipsic response perse, viz, withholding water reinforcement of the leverpress or the lick that is usually followed by water (Stein, 1964; Segal et al, 1965; Falk, $1966 \mathrm{a}, \mathrm{b})$. Extinction of an inducing schedule is a procedure often used in the study of alcohol self-administration to assess the reinforcing property of ethanol (Meisch \& Thompson, 1971; Freed et al, 1971). The present study was designed to contribute to the understanding of the basic polydipsic phenomenon by assessing the effects of chronic extinction of the inducing schedule on schedule-induced polydipsic behavior. \section{SUBJECTS}

The Ss were three male albino Holtzman rats maintained at approximately $80 \%$ of their free feeing weights, with water available at all times in the home cage except as noted below. Ss were individually housed in a constantly illuminated temperature-controlled room. They were approximately 3 months old at the start of the experiment.

*This research was supported in part by USPHS Research Grant MH 15349 to the University of Minnesota.

$\leftarrow$ Predoctoral trainee, USPHS Training Grant MH 08565 .

\section{APPARATUS}

The apparatus consisted of three identical Gerbrands rat operant conditioning chambers, each equipped with two levers, a food magazine, and a dipper mechanism. The levers were separated by the reinforcement mechanisms, with the food magazine to the left of the dipper. Reinforcers were $45-\mathrm{mg}$ Noyes pellets and $4 \mathrm{sec}$ access to a $.3 \cdot \mathrm{ml}$ dipper of water. Chambers were located in separate ventilated sound-attenuated enclosures. White noise was present at all times in the room. Programming and data recording were done automatically by electromechanical equipment located in another room.

\section{PROCEDURE}

The Ss were reduced to $80 \%$ of their free feeding weights and maintained at approximately those weights for the duration of the experiment. The subjects were $12 \mathrm{~h}$ water-deprived for the first four training sessions, during which 45-mg Noyes pellets were available for each response on the left lever (CRF) and 4-sec access to a . 3-ml dipper of water was available for each response on the right lever. This is the only time the Ss were water-deprived. On the fifth day, the food schedule was changed so that a pellet was delivered following the first response of the session on the left lever, subsequently, only the first response following a previously reinforced response of greater than a specified number of seconds was reinforced (FI n sec). Table 1 lists all schedule values and gives the number of sessions at each value. A 4-sec changeover delay from water to food responding was added at the FI $32-\mathrm{sec}$ parameter value. This was done to maximize the independence of water and food responding, and this procedure remained in effect for the duration of the experiment. Water was available on CRF during the duration of the experiment. Food sessions were terminated after 60 pellet deliveries; extinction sessions were run for $2 \mathrm{~h}$ $6 \mathrm{~min}$. After 10 sessions at FI $128 \mathrm{sec}$, the food dispenser was electronically disconnected and left lever responses had no programmed effect (Ext 1). This condition remained until the total number of food lever responses for the session declined to $1 \%$ or less of the mean of the last 3 days at FI $128 \mathrm{sec}$ ( 3 days for all three rats). The FI food contingency was reinstated (see Table 1 for parameter values and number of sessions) for 10 days (FI $128 \mathrm{sec}$ ), followed by 3 days of extinction. Although water was always available on CRF, the delivery time of the dipper mechanism allowed for the completion of two responses before the dipper reached the aperture of the water magazine. This led to ratios of 1.1-1.6 water responses per dipper presentation for different individuals. The ratio for each $\mathrm{S}$ remained stable across parameter values; however, it is felt that the number of dipper presentations more accurately reflects the polydipsic behavior. Data are presented as number of dipper presentations.

RESULTS AND DISCUSSION

The number of dipper presentations per quarter $(31.5 \mathrm{~min})$ of daily extinction sessions is shown in Fig. 1 . Dipper presentations fell markedly on Day 1 of Ext 1 to $17 \%-68 \%$ of baseline. Total dipper presentation percentages for Day 1 of Ext 2 are closely related to the percentage for Ext 1, the difference being 1-11 percentage points. Total dipper presentation percentages for Day 2 of Ext 1 is essentially unchanged from Day 1 of Ext 1. The percentage for Day 2 of Ext 2, however, differs markedly from Day 1 of Ext 2. This effect is consistent across all Ss. On Day 3 of Ext 1, the total dipper presentations fell to $6 \%-11 \%$ of baseline. Day 3 of Ext 2 parallels the above for two of the three Ss, $R 24$ and R 27; however, the Day 3 Ext 2 total percentage for these two $S$ s is approximately $1 / 2-1 / 3$ of their Day 3

Table 1

Parameter Values of the Food Schedule and Number of Sessions at Each Value for All Phases of the Experiment

\begin{tabular}{lc}
$\begin{array}{c}\text { Schedule } \\
\text { Value }\end{array}$ & $\begin{array}{c}\text { Number } \\
\text { of Sessions }\end{array}$ \\
\hline CR F & 4 \\
FI 16 Sec & 4 \\
FI 32 Sec & 4 \\
FI 64 Sec & 4 \\
FI 1 28 Sec & 10 \\
Ext 1 & 3 \\
FI 64 Sec & 4 \\
FI 32 Sec & 4 \\
FI $128 \mathrm{Sec}$ & 10 \\
Ext 2 & 3 \\
\hline
\end{tabular}




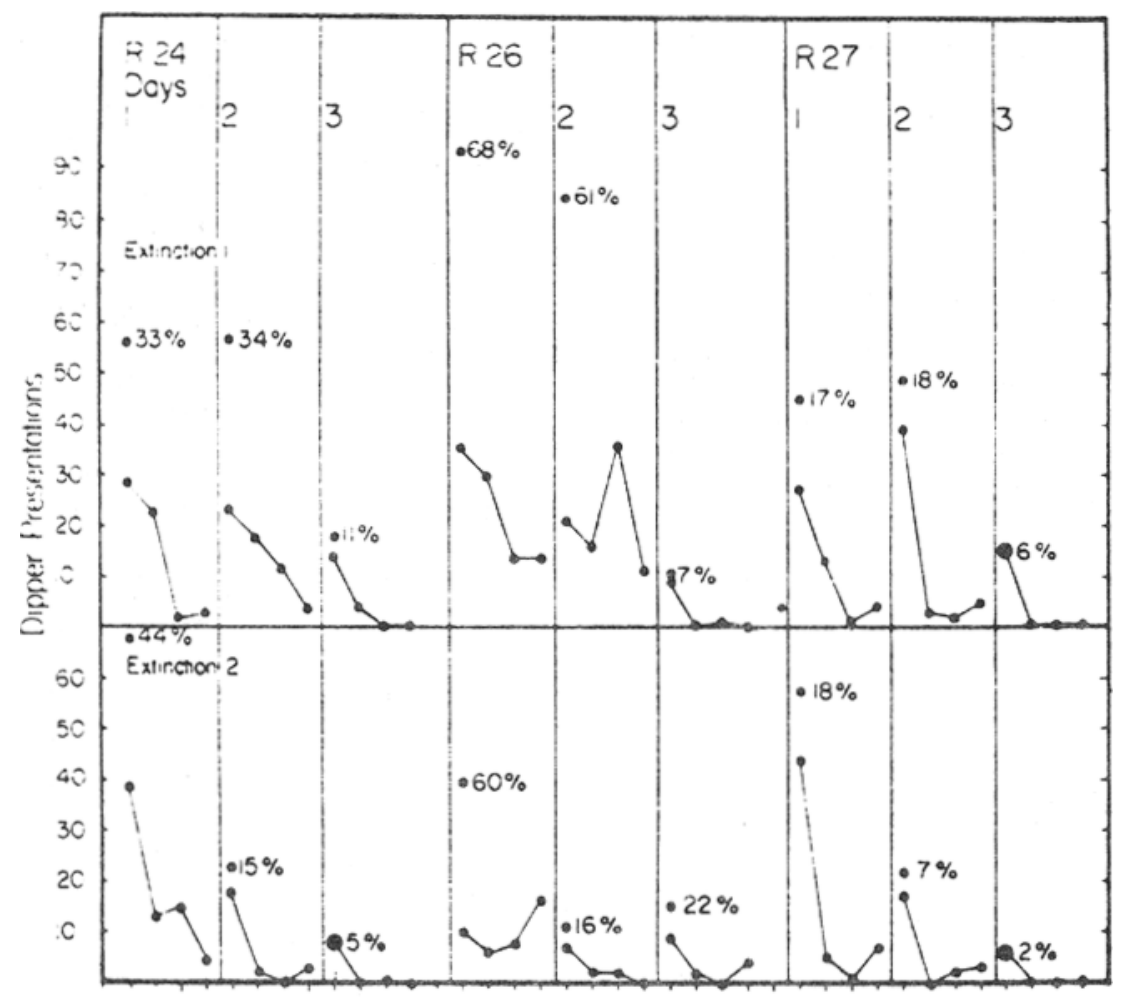

Successive Quarters of Sessions

Fig. 1. Number of dipper presentations per quarter $(31.5 \mathrm{~min})$ of three consecutive daily sessions of extinction of an FI 128-sec polydipsia-inducing food schedule. Ext 1 follows 10 sessions at FI $128 \mathrm{sec}$, and Ext 2 is a replication of Ext 1. Points above the daily quarter plots represent the total number of dipper presentations for the day below. Percentages next to the daily total points are percent of the mean number of dipper presentations of the last 3 days of the FI 128-sec baseline prior to that extinction phase.

Ext 1 percentage. Total dipper presentations for Day 3 of Ext 2 for R 26 did not fall from Day 2 of Ext 2, but rose from an absolute of 11 dipper presentations to 15 dipper presentations. This $\mathrm{S}$ differed from the other two in one more respect. The baseline polydipsia level prior to Ext 2 declined to approximately $1 / 2$ the previous baseline level. This may be seen by comparing Day 1 percentage values, which are very similar, to their respective ordinate values.

Most of the water responding occurred during the first quarter, R 26's data for Ext 1 being the exception. Whenever the first quarter responding of a subsequent extinction day exceeds the fourth quarter responding of a prior extinction day, spontaneous recovery has occurred. Comparing the first quarter dipper presentations of Day 2 with the fourth elimination of the behavior required three consecutive sessions for both the first and second periods of extinction. The second extinction period yielded fewer total responses and generally terminated with a lower rate of responding. The results of an earlier extinction study (Freed et al, 1970) indicated that two consecutive 1-h extinction sessions produced virtual elimination of water consumption. The difference between this figure and that found above may be due to the differences in parameter values, hours of previous training, or method of water presentation (spout vs dipper). The spontaneous recovery of polydipsia observed in this study is not unique but, rather, is probably universal to all studies of extinction of a polydipsia-inducing schedule; however, previous studies were not designed to observe this phenomenon.

\section{REFERENCES}

CLARK F C Some observations on the adventitious reinforcement of drinking under food reinforcement. Journal of the Experimental Analysis of Behavior, 1962. 5. 61-63.

FALK. J. L. Production of polydipsia in normal rats by an intermittent food schedule. Science, 1961, 133, 195-196.

FALK. J. L. The motivational properties of schedule induced polydipsia. Journal of the Experimental Analysis of Behavior, $1966 \mathrm{a}, 9,19-25$.

FALK, J. L. Schedule induced polydipsia as a function of fixed interval length. Journal of the Experimental Analysis of Behavior, $1966 \mathrm{~b}, 10.199-206$.

FREED, E. X., CARPENTER, J. A., \& HYMOWITZ. N. Acquisition and extinction of schedule induced polydipsic consumption of alcohol and water. Psychological Reports, 1970, 26, 915-922.

quarter of Day 1, or the first quarter of Day 3 with the fourth quarter of Day 2, reveals that spontaneous recovery of polydipsia occurred whenever numerically possible, i.e., the two instances that do not show spontaneous recovery occur on Day 3 of Ext 1 and Day 2 of Ext 2 for R 26 . On both of these occasions, the total daily dipper presentations fell short of the number of dipper presentations during the fourth quarter of the preceeding extinction day, thereby making it mathematically impossible to demonstrate spontaneous recovery, even if all the dipper presentations of the days in question occurred during the first quarter.

The results of the present study revealed the time course for the elimination of polydipsic behavior upon discontinuance of the food schedule that induced it. Virtual
HAWKINS, T. D. Pellet frequency: Effects on schedule induced polydipsia and variable interval barpress performance. Paper presented at the Psychonomic Society meeting, Chicago, 1967.

MEISCH, R. A., \& THOMPSON, T. Ethanol intake in the absence of concurrent food re in forcement. Psychopharmacologia (Berlin), 1971, 22, 72-79.

ROSENBLITH. J. Z. Poly dipsia induced in the rat by a second order schedule. Joumal of the Experimental Analysis of Behavior, 1970, 14, 139-144.

SEGAL, E. F., ODEN. D. L., \& DEADWYLER, S. A. Determinants of polydipsia: IV. Free reinforcement schedules. Psy chonomic Science, 1965, 3 . 11-12.

SCHAEFFER, R. W., \& DIEHL, J. C. Collateral water drinking in rats maintained on $F R$ food reinforcement schedules. Psychonomic Science, 1966. 4 , 257-258.

STEIN, L. Excessive drinking in the rat: Superstition or thirst? Journal of Comparative \& Physiological Psychology, $1964,58,237-242$. 